\title{
Forensic intoxication with clobazam: HPLC/DAD/MSD analysis
}

\author{
Paula Proença ${ }^{a, *}$, Helena Teixeiraa, ${ }^{a, b}$ João Pinheiro ${ }^{a}$, Estela P. Marques ${ }^{a, b}$, \\ Duarte Nuno Vieira ${ }^{\mathrm{a}, \mathrm{b}}$ \\ ${ }^{\mathrm{a}}$ National Institute of Legal Medicine-Delegation of Coimbra, Largo da Sé Nova, 3000-213 Coimbra, Portugal \\ ${ }^{\mathrm{b}}$ Faculty of Medicine, University of Coimbra, Largo da Sé Nova, 3000-213 Coimbra, Portugal
}

Received 20 November 2003; accepted 1 March 2004

Available online 28 May 2004

\begin{abstract}
Clobazam $\left(\right.$ Castillium $^{\mathbb{R}}$, Urbanil $^{\mathbb{R}}$ ), a benzodiazepine often used as an anxiolytic and in the treatment of epilepsy, is considered a relatively safe drug. The authors present a fatal case with a 49-year-old female, found dead at home. She had been undergoing psychiatric treatment and was a chronic alcoholic. The autopsy findings were unremarkable, except for multivisceral congestion, steatosis and a small piece of a plastic blister pack in the stomach. Bronchopneumonia, bronchitis and bronchiolitis were also diagnosed. Anhigh-performance liquid chromatography (HPLC)/diode array detector (DAD)/mass spectrometry detection (MSD) with electrospray method was developed in order to detect, confirm and quantify clobazam in the post-mortem samples. In the chromatographic separation, a reversed-phase column $\mathrm{C}_{18}(2.1 \times 150 \mathrm{~mm}, 3.5 \mu \mathrm{m})$ was used with a mobile phase of methanol and water, at a $0.25 \mathrm{ml} / \mathrm{min}$ flow rate. Carbonate buffer $(\mathrm{pH} 10.5)$ and $20 \mu \mathrm{l}$ of prazepam $(100 \mu \mathrm{g} / \mathrm{ml})$ as internal standard were added to the samples. A simple and reliable liquid-liquid extraction method for the determination of clobazam in post-mortem samples was described. Calibration curves for clobazam were performed in blood, achieving linearity between 0.01 and $10 \mu \mathrm{g} / \mathrm{ml}$ and a detection limit of $1.0 \mathrm{ng} / \mathrm{ml}$. The clobazam concentration found in post-mortem blood was $3.9 \mu \mathrm{g} / \mathrm{ml}$, higher than the reported therapeutic concentration $(0.1-0.4 \mu \mathrm{g} / \mathrm{ml})$. The simultaneous acquisition by photodiode array detection and mass spectrometry detection results allowed benzodiazepines to be identified with sufficient certainty.

An examination of all the available information suggested that death resulted from respiratory depression due to clobazam toxicity.

(C) 2004 Elsevier Ireland Ltd. All rights reserved.
\end{abstract}

Keywords: Forensic intoxication; Clobazam; HPLC/DAD/MSD

\section{Introduction}

Benzodiazepines are among the most widely prescribed drugs and are used in the treatment of stress, anxiety-induced depression, panic and sleep disorders, muscle spasms, alcohol withdrawal and seizures [1]. Unfortunately, they are often subject to overdose in suicide attempts and are frequently encountered in emergency intoxication episodes and drugs-of-abuse testing.

Clobazam $\left(\right.$ Castillium $^{\mathbb{R}}$, Urbanil $^{\mathbb{R}}$ ) is a new antiepileptic drug effective with most varieties of seizures and epilepsies

\footnotetext{
* Corresponding author. Tel.: +351-239854230; fax: $+351-239820549$.

E-mail address: paulaproenca@dcinml.mj.pt (P. Proença).
}

in both short-term and long-term treatment [2,3]. It is the first 1,5-benzodiazepine that has nitrogen atoms in positions 1 and 5 of the heterocyclic ring, whose chemical structure was designed to give it a different pharmacological profile from that of 1,4-benzodiazepines [4]. Clobazam has been shown to be a valuable addition to currently available regiments when used alone in the treatment of acute alcohol withdrawal, especially during the initial period where anxiety and insomnia related symptoms are at their peak [5]. The major metabolic pathway of this benzodiazepine in humans involves removal of the $\mathrm{N}-1$ methyl group, resulting in the formation of the main pharmacologically active metabolite desmethylclobazam [6-8] (Fig. 1).

Several methods for the determination of clobazam have been published. Immunoassay [8] approaches remain 


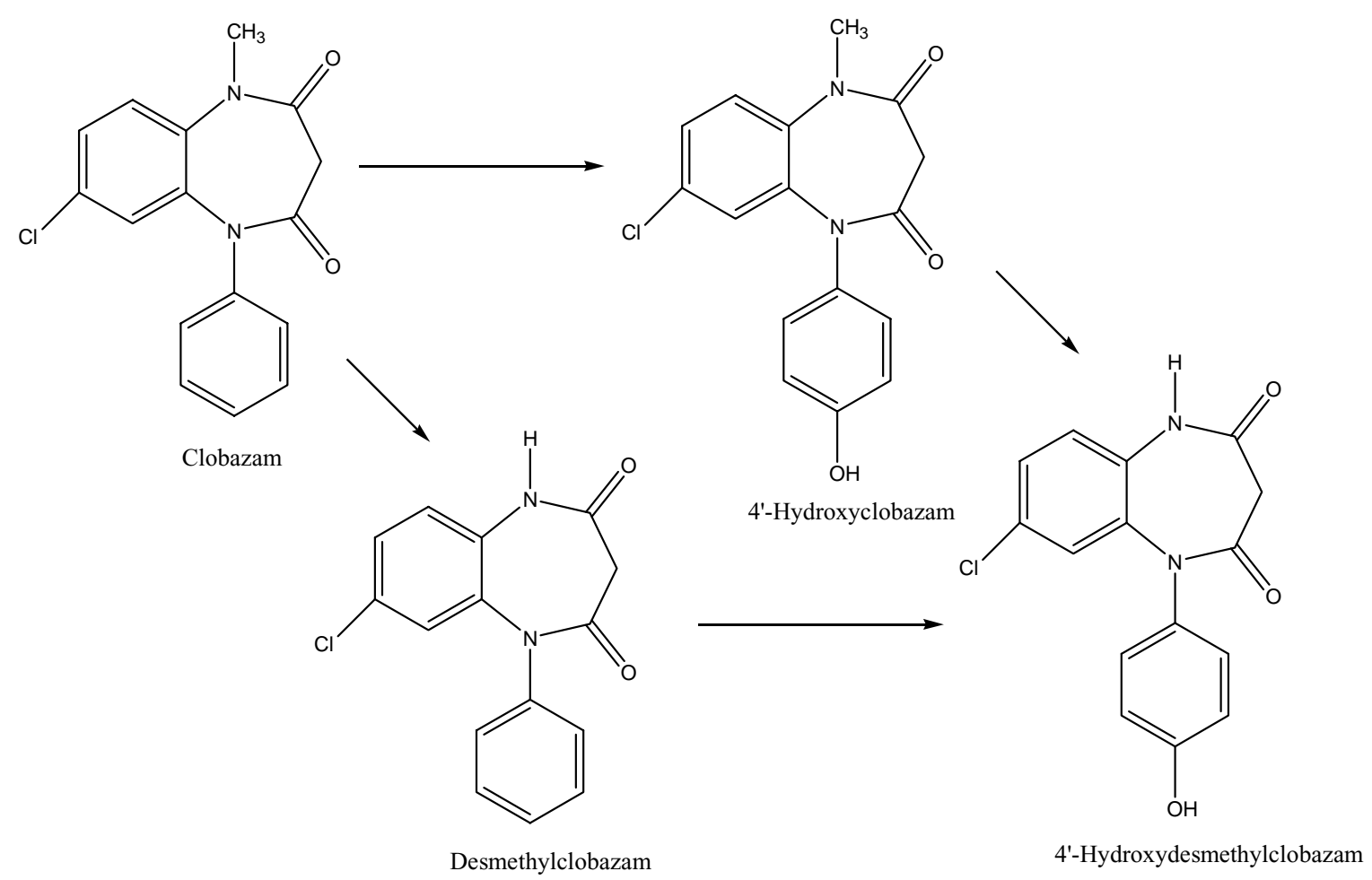

Fig. 1. Metabolic pathways for clobazam.

extremely useful for initial screening. Although gas chromatography [9] is much more sensitive and specific, it requires derivatization procedures and thermolabile benzodiazepines are not suited to this technique. As a consequence high-performance liquid chromatography (HPLC) [10-19] and more recently, the coupling of HPLC with mass spectrometry (MSD) [20,21] provides a useful and robust technique for the analysis of benzodiazepines.

In this study, we report a fatal case involving clobazam in high concentration and the identification of its metabolite in the post-mortem blood sample. An HPLC/diode array detector (DAD)/MSD with an electrospray source in positive mode was developed in order to detect, confirm and quantify this benzodiazepine in the post-mortem samples.

\section{Case report}

A 49-year-old Caucasian female was found dead by her husband, at home, lying on the floor of the bathroom. The forensic pathologist was called to perform a scene examination and to verify the time of death. Traces of vomit with no obvious smell and diarrhoea were present. Many different drugs were found in her bedroom: clobazam, bromazepam, zolpidem, fluoxetine, ranitidine and dissulfiram. She had been undergoing long term psychiatric treatment and had a history of chronic alcoholism. On the day of her death she had been seen at the hospital, by her psychiatrist.
Autopsy was performed 3 days after death at the Forensic Pathology Service of the Coimbra Delegation of the National Institute of Legal Medicine (NILM). At the autopsy, an external examination revealed rash, eye congestion, abrasions on the right lower extremity and echymoses on the upper and lower extremities of the abdomen. The internal examination revealed signs of visceral congestion, hepatic steatosis and a small piece of a plastic blister pack in the stomach. Microscopic findings showed focus of pneumonia, bronchitis and bronchiolitis, with intra-alveolar hemorrhages and focal mild pulmonary edema. The kidneys showed chronic tubular nephritis. Samples of the blood, stomach, liver and kidney were taken for toxicological analysis.

\section{Materials and methods}

\subsection{Chemicals}

Clobazam and prazepam (used as internal standard) were supplied by Sigma-Aldrich-Chemie GmbH (Steinheim, Germany). Each standard compound was dissolved in methanol $(1 \mathrm{mg} / \mathrm{ml})$ and stored at $4{ }^{\circ} \mathrm{C}$. All solvents were analytical or HPLC grade and were purchased from E. Merck (Darmstadt, Germany). Water was purified by a Milli-Q system obtained from Millipore (Molsheim, France). The carbonate buffer ( $\mathrm{pH}$ 10.5) was prepared by 
dissolving $4.2 \mathrm{~g}$ of $\mathrm{NaHCO}_{3}$ into a $100 \mathrm{ml}$ volumetric flask and brought to volume with demonised water. The buffer was stored refrigerated $\left(4{ }^{\circ} \mathrm{C}\right)$. The mobile phase was filtered with a $0.20 \mu \mathrm{m}$ Schleicher \& Schuell filter and degassed in an ultrasonic bath for $15 \mathrm{~min}$ just before use.

\subsection{Instrumentation}

The chromatographic system used was a Waters 2695 Alliance System and a Symmetry ${ }^{\mathbb{B}} \mathrm{C}_{18}$ reversed-phase column $(2.1 \mathrm{~mm}$ i.d. $\times 150 \mathrm{~mm}, 3.5 \mu \mathrm{m})$. A methanol/water mobile phase gradient was used for compound separation, programmed to be held at $55 \%$ methanol for $5 \mathrm{~min}$ and then increased to $65 \%$ over $12 \mathrm{~min}$, followed by a hold at $55 \%$ methanol for $5 \mathrm{~min}$. A $5 \mathrm{~min}$ reconditioning time was allowed before the next injection. The total run time was $30 \mathrm{~min}$ at a flow rate of $0.250 \mathrm{ml} / \mathrm{min}$. The separation was performed at $25^{\circ} \mathrm{C}$. The injection volume was $5 \mu \mathrm{l}$.

For detection, a Waters 996 photodiode array detector was used, operated on a $210-400 \mathrm{~nm}$ wavelength scan with a $1.2 \mathrm{~nm}$ resolution. The UV absorbance was measured at $254 \mathrm{~nm}$. Instrument control, data acquisition and processing were achieved by the use of Waters Empower software (Milford, MA).

Mass spectrometry detection was carried out by a Waters ZQ 2000 mass spectrometer with an electrospray ionisation (ESI) performed in positive mode. Full-scan spectra were recorded from $m / z, 180-380$, at a scan time of $0.5 \mathrm{~s}$ and an interscan delay of $0.1 \mathrm{~s}$. The mass spectra were represented in centroid mode. The other main instrument settings were: capillary voltage $3.5 \mathrm{kV}$; cone voltage $45 \mathrm{~V}$; extractor $3 \mathrm{~V}$; ion energy 0.1 ; source temperature $80{ }^{\circ} \mathrm{C}$; desolvation temperature $150{ }^{\circ} \mathrm{C}$; cone gas $\left(\mathrm{N}_{2}\right)$ flow rate $50 \mathrm{l} / \mathrm{h}$ and desolvation gas $\left(\mathrm{N}_{2}\right)$ flow rate $250 \mathrm{l} / \mathrm{h}$.

Quantitation made use of the selected ion-recording mode (SIR) using the most abundant characteristic ion $[M+\mathrm{H}]^{+}$, $\mathrm{m} / \mathrm{z}, 301$ and the fragment ion, $\mathrm{m} / \mathrm{z} 259$, for confirmation.

The other drugs (zolpidem, fluoxetine, ranitidine and disulfiram) were analysed by gas chromatography with a mass spectrometry detector [22]. Ethanol was quantified by conventional gas chromatography with a flame ionisation detector and a headspace system (GC/FID/HS), using npropanol as internal standard.

\subsection{Sample preparation}

Control and calibration samples were prepared by spiking drug-free post-mortem blood samples with standard solutions.

A $1 \mathrm{ml}$ aliquot of whole blood or $1 \mathrm{~g}$ of tissue (cut in small pieces) was spiked with $20 \mu \mathrm{l}$ of internal standard (prazepam, $100 \mu \mathrm{g} / \mathrm{ml})$. Then $0.5 \mathrm{ml}$ of carbonate buffer $(50 \mathrm{mM})$ was added to bring the sample to alkaline conditions $(\mathrm{pH}$ $10.5)$, followed by agitation in a vortex mixer.

Two liquid-liquid extractions were performed, one with $6 \mathrm{ml} \mathrm{n}$-hexane: ethyl acetate $(7: 3, \mathrm{v} / \mathrm{v})$ and the other with
$6 \mathrm{ml}$ n-hexane:2-propanol (99:1, v/v) in a rotamix device for $15 \mathrm{~min}$. After centrifugation at $3000 \mathrm{rpm}$ for $20 \mathrm{~min}$. The combined supernatant was evaporated to dryness under a slow stream of nitrogen, at $40{ }^{\circ} \mathrm{C}$.

The dried extracts were reconstituted with $250 \mu$ methanol and an aliquot $(5 \mu \mathrm{l})$ was injected into the HPLC/DAD/ MSD system.

\section{Results and discussion}

Calibration curves were linear from 0.01 to $10 \mu \mathrm{g} / \mathrm{ml}$. Eight calibrators were used to generate a standard curve, in triplicate. The calibration curves were constructed from peak areas using the SIR mode and showed a linear relationship for clobazam with a coefficient of correlation of 0.9988 . Due to the high clobazam concentration found in the presented case, it was necessary to develop the method with a larger concentration range.

The detection limit (LOD) was estimated from extracted blood samples spiked with decreasing concentrations of clobazam, where the response, of the protonated molecular ion in SIR mode, was equivalent to three times the baseline noise. The limit of quantitation (LOQ) was the lowest amount of analyte in a sample that could be quantitatively determined with a suitable precision and accuracy (coefficients of variability, CV < 20\%).The LOD for clobazam was $1.0 \mathrm{ng} / \mathrm{ml}$ and LOQ, was $10.0 \mathrm{ng} / \mathrm{ml}$.

Extraction recovery was determined by comparing the representative peak areas of extracted drug-free blood spiked before extraction with the peak area of drug-free blood fortified before injection at the same concentration. Table 1 shows that the calculated extraction efficiencies for clobazam ranged from 76 to $99 \%$. Intra-day and interday coefficients of variability values were determined by replicate analyses $(n=5)$ of blood sample aliquots, either on the same run (intra-day) or on separate days (inter-day). The method proved to be precise for clobazam, both in terms of intra-day and inter-day analysis, with coefficients of variation of less than $10 \%$.

The chromatographic method described had a run time of $30 \mathrm{~min}$, and clobazam, was detected at a retention time of $6.7 \mathrm{~min}$.

Table 1

Validation data of recovery and precision for clobazam in spiked blood samples

\begin{tabular}{lllll}
\hline $\begin{array}{l}\text { Concentration level } \\
(\mu \mathrm{g} / \mathrm{ml})\end{array}$ & $\begin{array}{l}\text { Recovery } \\
(\%)\end{array}$ & $\begin{array}{l}\text { Intra-day } \\
\mathrm{CV}(\%)\end{array}$ & $\begin{array}{l}\text { Inter-day } \\
\mathrm{CV}(\%)\end{array}$ & $n$ \\
\hline 0.05 & 76 & 8.3 & 9.7 & 5 \\
0.1 & 88 & 5.7 & 4.5 & 5 \\
1 & 97 & 3.1 & 4.9 & 5 \\
2 & 92 & 3.4 & 5.3 & 5 \\
5 & 99 & 2.2 & 3.5 & 5 \\
\hline
\end{tabular}


Since the procedure was found to be sensitive, selective and reproducible, the developed method was applied to the fatal case presented here. The toxicological analysis showed clobazam concentrations of $3.9 \mu \mathrm{g} / \mathrm{ml}$ in the blood, $1.1 \mu \mathrm{g} / \mathrm{g}$ in the stomach, $2.4 \mu \mathrm{g} / \mathrm{g}$ in the liver and $5.3 \mu \mathrm{g} / \mathrm{g}$ in the kidney. Neither ethanol or other drugs were detected in the post-mortem samples.

Quantitation employed the selected ion-recording mode using the most abundant characteristic ion, $\mathrm{m} / \mathrm{z} 301$, and the fragment ion, $m / z 259$, for confirmation. The fragmentation voltage from 20 to $70 \mathrm{~V}$ was also investigated. On increasing the fragmentation voltage, the clobazam fragmentation pattern became more complex. The abundance of the molecular ion $[M+\mathrm{H}]^{+}$for this benzodiazepine decreased with higher fragmentation voltages $(>40 \mathrm{~V})$ and the characteristic ions appeared. Depending on the compound structure, the characteristic ions appeared at different fragmentation voltages [21]. The results of the positive electrospray ionisation mass spectrum of clobazam with a cone voltage of 40 and $60 \mathrm{~V}$, obtained after injection of extracted blood samples, are shown in Fig. 2.

The blood concentration of clobazam, in the fatal case, was $3.9 \mu \mathrm{g} / \mathrm{ml}$, which is much higher than the reported therapeutic level $(0.1-0.4 \mu \mathrm{g} / \mathrm{ml})$ [23]. However, it is a fact that in assessing whether benzodiazepines have contributed significantly to a death, it is essential to have access to a full medical history and a detailed record of the autopsy findings [24]. The main pharmacologically active metabolite desmethylclobazam was also detected (Fig. 3), but was not quantified due to the lack of the corresponding standard. However, given that it is obviously an important metabolite which is rapidly formed from clobazam and usually the major species present, even in overdoses, the clobazam
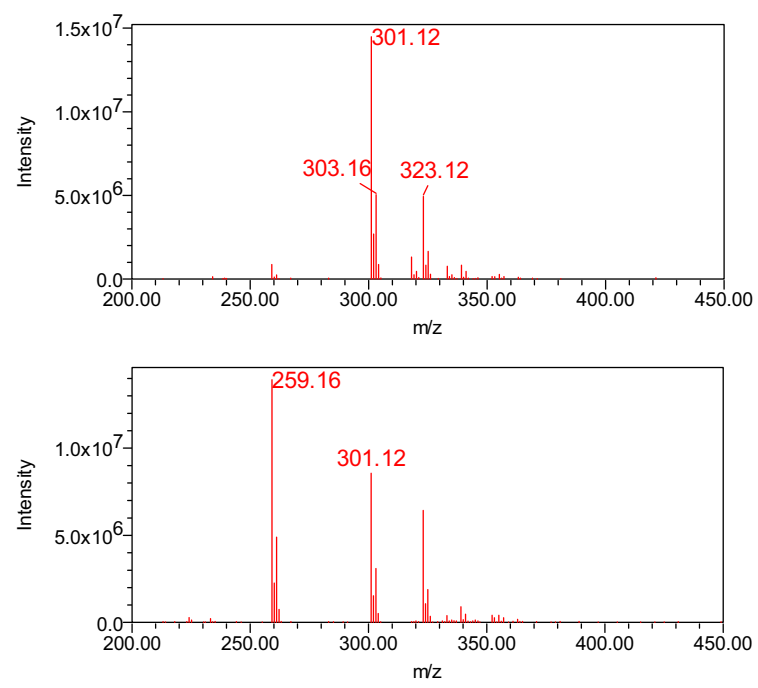

Fig. 2. Positive ESI mass spectrum of clobazam: cone voltage of 40 and $60 \mathrm{~V}$.

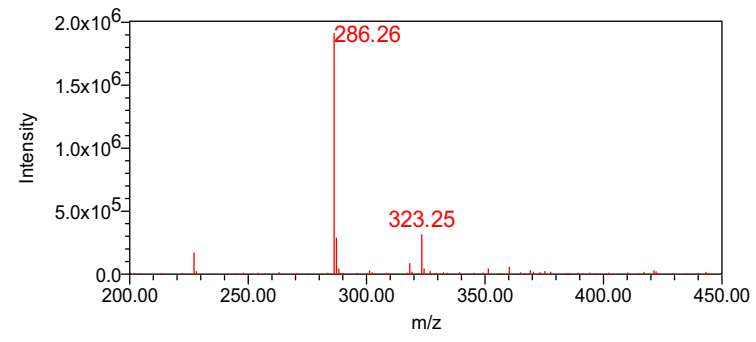

Fig. 3. Positive ESI mass spectrum of desmethylclobazam: cone voltage of $65 \mathrm{~V}$.

concentration found in blood is sufficient to allow some conclusions to be reached concerning intoxication since the authors [24] who have reported the only documented case of clobazam intoxication achieved a lower concentration of clobazam and did not even refer to the detection of its metabolite. These authors reported a fatal case caused by an overdose of clobazam and diazepam, in a man who developed bronchopneumonia, with a clobazam concentration of $1.5 \mu \mathrm{g} / \mathrm{ml}$ in post-mortem blood. This is also a case where other drugs, including ethanol, did not contribute to the death. It is particularly significant to note that bronchopneumonia was also found in our case. Indeed the development of bronchopneumonia is recognised as being associated with immobility and coma, whether drug induced or otherwise. [24,25], and thus, it is important to emphasise the fact that the sedatives may contribute to the development of pneumonia, which can have the additional effect of producing a toxaemia. On the other hand, some authors [24] also claim that some of the individuals shown to have bronchopneumonia in association with the injection of benzodiazepines prior to death, suggests that there is a period of survival following drug injection and excretion of drugs may occur resulting in the lowering of post-mortem drug concentrations.

Clobazam was also detected in other tissues, which could reveal a possible re-distribution phenomenon. Postmortem drug redistribution has been reported for many drugs [2631], but few have assessed whether benzodiazepines in general undergo post-mortem redistribution [26,27,29-31]. The process of redistribution can affect the concentration of all analytes in post-mortem blood as a result of the disruption of cellular membranes, causing alterations of drug concentrations within tissue elements and diffusion from one tissue to another. This process is particularly significant for drugs with high lipid solubility or high tissue concentrations relative to blood [31]. In cases in which significant residues were still present in gastric contents there was no apparent increase in blood concentrations of nitrobenzodiazepines and metabolites. Therefore, some authors concluded that post-mortem diffusion into peripheral blood is not a confounding factor in the interpretation of benzodiazepines concentrations $[30,32]$. 


\section{Conclusion}

As a result of the background circumstances and the autopsy findings, it was concluded that clobazam was responsible for the death, probably by respiratory depression. When poisoning occurs, respiratory depression and pneumonia are present $[24,26]$, as seen in this case, including bronchopneumonia, bronchitis and bronchiolitis.

\section{References}

[1] J.G. Hardman, L.E. Limbird, P.B. Molinoff, R.W. Ruddon, A.G. Gilman, Goodman and Gilman's The Pharmacological Basis of Therapeutics, ninth ed., McGraw-Hill, 1996.

[2] C. Remy, Clobazam in treatment of epilepsy: a review of the literature, Epilepsia 35 (1994) 88-91.

[3] H. Nakajima, A pharmacological profile of clobazam (Mystan), a new antiepileptic drug, Nippon Yakurigaku Zasshi 118 (2001) 117-122.

[4] H. Kuch, Clobazam: chemical aspects of the 1,4 and 1,5benzodiazepines, Br. J. Clin. Pharmacol. 7 (Suppl. 7) (1979) $17-21$.

[5] P.K. Mukherjee, A comparison of the efficacy and tolerability of clobazam and chlordiazepoxide in the treatment of acute withdrawal from alcohol in patients with primary alcoholism, J. Int. Med. Res. 11 (1983) 205-211.

[6] M. Volz, O. Christ et al., Kinetics and metabolism of clobazam in animals and man, Br. J. Clin. Pharmacol. 7 (1979) 41-50.

[7] A.C. Moffat, J.V. Jackson, M.S. Moss, Clarke's isolation and identification of drugs in pharmaceuticals, body fluids, and post-mortem materials, The Pharmaceutical Press, London, 1986, pp. 475-476.

[8] W. Huang, D.E. Moody, Immunoassay detection of benzodiazepines and benzodiazepine metabolites in blood, J. Anal. Toxicol. 19 (1995) 333-342.

[9] D.F. LeGatt, D.P. McIntosh, Clobazam and norclobazam quantification in serum by capillary gas chromatography with electron-capture detection, Clin. Biochem. 26 (1993) 159-163.

[10] J. Knapp, P. Bokník et al., Quantification of clobazam in human plasma using high-performance liquid chromatography, J. Chromatogr. Sci. 37 (1999) 145-149.

[11] C. Lacroix, F. Wojciechowski et al., Monitoring of benzodiazepines (clobazam, diazepam and their main active metabolites) in human plasma by column-switching highperformance liquid chromatography, J. Chromatogr. 617 (1993) 285-290.

[12] K.K. Akerman, Analysis of clobazam and its active metabolite norclobazam in plasma and serum and urine using HPLC/DAD, Scand. J. Clin. Lab. Invest. 56 (1996) 609-614.

[13] J.M. Streete, D.J. Berry et al., The analysis of clobazam and its metabolite desmethylclobazam by high-performance liquid chromatography, Ther. Drug Monit. 13 (1991) 339-344.

[14] A. Brachet-Liermain, C. Jarry et al., Liquid chromatography determination of clobazam and its major metabolite $\mathrm{N}$ desmethylclobazam in human plasma, Ther. Drug Monit. 4 (1982) 301-305.

[15] A. Bolmer, F. Tagliaro et al., Optimised determination of clobazam in human plasma with extraction and high-performance liquid chromatography analysis, J. Chromatogr. B Biomed. Sci. Appl. 750 (2001) 177-180.

[16] P.K. Kunicki, Simples and sensitive high-performance liquid chromatography method for the determination 1,5-benzodiazepine clobazam and its active metabolite N-desmethylclobazam in human serum and urine with application to 1,4benzodiazepines analysis, J. Chromatogr. B Biomed. Sci. Appl. 750 (2001) 41-49.

[17] W.R. Gazdzik, J. Podlesny et al., HPLC method for simultaneous determination of clobazam and N-desmethylclobazam in human serum, rat serum and rat brain homogenates, Biomed. Chromatogr. 3 (1989) 79-81.

[18] M. Wilhelm, H. Battista et al., HPLC with simultaneous UV and reductive electrochemical detection at the hanging mercury drop electrode: a highly sensitive and selective tool for the determination of benzodiazepines in forensic samples, J. Anal. Toxicol. 25 (2001) 250-257.

[19] M.D. Robertson, O.H. Drummer, High-performance liquid chromatographic procedure for the measurement of nitrobenzodiazepines and their 7-amino metabolites in blood, J. Chromatogr. 667 (1995) 179-184.

[20] A.M. Oxley, T.D. Lee, et al., A method for the simultaneous determination of clobazam and desmethylclobazam in postmortem blood by HPLC-MS-MS, in: Proceedings of the 40th Annual Meeting of the International Association of Forensic Toxicologists, 2002.

[21] H. Yuan, Z. Mester et al., Automated in tube solid-phase microextration couple with liquid chromatography-electrospray ionisation mass spectrometry for the determination of selected benzodiazepines, J. Anal. Toxicol. 24 (2000) 718-725.

[22] E.P. Marques, F. Gil, P. Proença et al., Analytical method for the determination of strychnine in tissues by gas chromatography/mass spectrometry: two case report, Forensic Sci. Int. 110 (2000) 145-152.

[23] Therapeutic and Toxic Drug Concentrations List, http:// www.tiaft.org/tmembers/ttv/ttv_ab.html.

[24] O.H. Drummer, D.L. Ranson, Sudden death and benzodiazepinas, Am. J. Forensic Med. Patholog. 17 (1996) 336-342.

[25] http://www.inchem.org/documents/pims/pharm/pim921.html.

[26] D.J. Pounder, G.R. Jones, Post-mortem drug redistribution-a toxicological nightmare, Forensic Sci. Int. 45 (1990) 253-263.

[27] R.W. Prouty, W.H. Jones, The forensic science implications of site and temporal influences on post-mortem blood-drug concentrations, J. Forensic Sci. 35 (1990) 243-270.

[28] G.R. Jones, Site dependent differences in the blood levels of some drugs, in: Proceedings of the 24th International Meeting of the International Association of Forensic Toxicologists, 1988.

[29] D.J. Pounder, The nightmare of post-mortem drug changes, Legal Med. Ann. 105 (1992) 87-91.

[30] M.D. Robertson, O.H. Drummer, Postmortem distribution and redistribution of nitrobenzodiazepines in man, J. Forensic Sci. 43 (1998) 9-13.

[31] O.H. Drummer, J. Gerostamoulos, Postmortem drug analysis: analytical and toxicological aspects, Ther. Drug Monit. 24 (2002) 199-209.

[32] T. Hilberg, A. Bugge et et al., Diffusion as a mechanism of postmortem redistribution. An experimental study in rats, Int. J. Legal Med. 105 (1992) 87-91. 